8. Radonovich LJ, Cheng J, Shenal BV, Hodgson M, Bender BS. Respirator tolerance in health care workers. JAMA 2009; 301:36-38.

9. United States Department of Labor, Occupational Safety and Health Administration. Occupational safety and health standards: bloodborne pathogens, final rule. 29 CFR $\$ 1910.1030$. Washington, DC: United States Department of Labor, Occupational Safety and Health Administration, 2001.

10. National Institute for Occupational Safety and Health. Respirator testing and certification (non-CBRN)-FY08. 2008. Available at: http://www .cdc.gov/niosh/nas/ppt/QUADCharts08/PP21_FY08_QC.htm. Accessed January $7,2009$.

\section{Did CA-MRSA Bacteremia Exist in Taiwanese Patients With End-Stage Renal Disease?}

To the Editor-In a recently published study, Lin et al. ${ }^{1}$ attempted to distinguish between the clinical characteristics of patients infected with community-associated methicillin-resistant Staphylococcus aureus (CA-MRSA) and those of patients infected with healthcare-associated (HA-MRSA). The study population consisted of patients who were receiving peritoneal dialysis or hemodialysis. This fact contradicts the present definition for CA-MRSA, because MRSA detected in persons with healthcare-associated risk factors, such as dialysis, within 1 year before onset of MRSA infection is not considered to be community acquired. ${ }^{2}$

One of the other criteria adopted by Lin and colleagues for identifying CA-MRSA and HA-MRSA was staphylococcal cassette chromosome (SCC) mec typing. They identified MRSA strains with SCCmec types IV or V as community acquired and MRSA strains with SCCmec types II or III as healthcare acquired. The designation of the source of MRSA acquisition by means of SCCmec typing may be misleading. In 2007, researchers at National Taiwan University Hospital (Taipei, Taiwan), the site of the study by Lin et al,, ${ }^{1}$ reported that SCCmec type III predominated during 1999-2004, whereas SCCmec types IV and V predominated during $2005 .^{3}$ Others have also reported changes in the predominant SCCmec types over time. ${ }^{4}$ Therefore, this use of SCCmec typing may not be an accurate method for distinguishing between HA-MRSA and CA-MRSA.

\section{ACKNOWLEDGMENTS}

Potential conflicts of interest. All authors report no conflicts of interest relevant to this letter.

Hung-Chin Tsai, MD, PhD; Cheng-Len Sy, MD; Yao-Shen Chen, MD
From the Section of Infectious Diseases, Department of Medicine, Kaohsiung Veterans General Hospital, Kaohsiung (H.-C.T., C.-L.S., Y.-S.C.), and the National Yang-Ming University, Taipei (H.-C.T., C.-L.S., Y.-S.C.), Taiwan

Address reprint requests to Hung-Chin Tsai, MD, Section of Infectious Diseases, Department of Medicine, Kaohsiung Veterans General Hospital, 386 Ta-Chung 1st Road, Kaohsiung 813, Taiwan (hctsai1011@yahoo.com tw).

All authors contributed equally to the composition of this letter. Infect Control Hosp Epidemiol 2009; 30:718-718

(C) 2009 by The Society for Healthcare Epidemiology of America. All rights reserved. 0899-823X/2009/3007-0020\$15.00. DOI: $10.1086 / 598238$

\section{REFERENCES}

1. Lin CC, Wang JL, Lin CY, et al. Methicillin-resistant Staphylococcus aureus bacteremia in patients with end-stage renal disease in Taiwan: distinguishing between community-associated and healthcare-associated strains. Infect Control Hosp Epidemiol 2009; 30:89-92.

2. Nathwani $D$, Morgan $M$, Masterton RG, et al. Guidelines for UK practice for the diagnosis and management of methicillin-resistant Staphylococcus aureus (MRSA) infections presenting in the community. I Antimicrob Chemother 2008; 61:976-994.

3. Huang YH, Tseng SP, Hu JM, Tsai JC, Hsueh PR, Teng LJ. Clonal spread of SCCmec type IV methicillin-resistant Staphylococcus aureus between community and hospital. Clin Microbiol Infect 2007; 13:717-724.

4. Wisplinghoff $\mathrm{H}$, Ewertz B, Wisplinghoff $\mathrm{S}$, et al. Molecular evolution of methicillin-resistant Staphylococcus aureus in the metropolitan area of Cologne, Germany, from 1984 to 1998. J Clin Microbiol 2005; 43:5445-5451.

\section{Reply to Tsai et al.}

To the Editor-We agree with Tsai et al. ${ }^{1}$ that staphylococcal cassette chromosome (SCC) mec typing may not be sensitive enough and specific enough to accurately classify methicillinresistant Staphylococcus aureus (MRSA) infections as either healthcare associated (HA) or community associated (CA). In addition to the different molecular epidemiologic characteristics of CA-MRSA strains in Taiwan, the evidence of continued spread of CA-MRSA strains into hospital settings ${ }^{2-4}$ and the detection of SCCmec type IV in a HA-MRSA strain, namely EMRA-15 (ST22-IV), which is endemic in many hospitals throughout the world, lead to occasional confusion regarding the definitions of CA-MRSA and HA-MRSA infections. ${ }^{5,6}$ However, molecular epidemiological definitions based on SCCmec typing and phylogenetic analyses of the MRSA isolates are still regarded as the most reliable means for distinguishing between HA-MRSA and CA-MRSA strains. ${ }^{5}$ In fact, MRSA strains carrying different SCCmec types are biologically different. The rationale for defining isolates carrying SCCmec types IV and V as CA-MRSA is based on the relatively small size of its genetic components, which facilitates the survival of CA-MRSA in the community setting. ${ }^{7,8}$ On the contrary, antibiotic selective pressure and cross-transmission in the nosocomial setting contribute to the survival of MRSA isolates 